\title{
Interacción universidad y entorno: marco para el emprendimiento ${ }^{1}$
}

\section{Pedro Emilio Sanabria-Rangel}

Universidad Militar Nueva Granada, Bogotá (Colombia)

pedro.sanabria@unimilitar.edu.co
María Eugenia Morales-Rubiano

Universidad Militar Nueva Granada, Bogotá (Colombia) maria.morales@unimilitar.edu.co

\section{Carolina Ortiz-Riaga}

Universidad Militar Nueva Granada,

Bogotá (Colombia)

maria.ortiz@unimilitar.edu.co

\section{Resumen}

El documento desarrolla el tema de la relación universidad-entorno, especialmente en lo que se refiere a la vinculación con el sector productivo como parte del desarrollo de la función de extensión universitaria.

El propósito fundamental es proporcionar un marco de referencia para la creación o justificación de las unidades de emprendimiento universitarias como mecanismo de enlace para el desarrollo de la función extensión desde una de sus aproximaciones: el enfoque empresarial.

Como resultado, se evidencian los enfoques, los modelos, los mecanismos y las estrategias de interacción universidad-entorno; se realiza una reflexión sobre el emprendimiento como medio para dicha interacción y se muestran las condiciones estructurales que favorecen el emprendimiento universitario.

1 Artículo derivado del proyecto de la UMNG denominado Integración Universidad-Empresa-Estado, financiado con recursos de la UMNG y desarrollado por el Grupo de Estudios Contemporáneos en Contabilidad, Gestión y Organizaciones dentro de la línea de investigación en Estudios Contemporáneos en Gestión y Organizaciones.

Recepción: 2014-02-04 | Envío a pares: 2014-09-14 | Aceptación por pares: 2015-03-16 | Aprobación: 2015-04-04

Sanabria, P. E., Morales, M. E. y Ortiz Riaga C. (2015). Interacción universidad y entorno: marco para el emprendimiento. Educ. Educ. Vol. 18, No. 1, 111-134. DOI: 10.5294/edu.2015.18.1.7 


\title{
Palabras clave
}

Progreso académico, articulación educativa, gestión educativa, eficacia educativa (Fuente: Tesauro de la Unesco).

\section{Interaction between the University and the Environment: A Framework for Entrepreneurship}

\begin{abstract}
This article looks at the university-environment relationship, particularly in terms of links to the productive sector as part of the development of the extension function of the university.

The primary purpose is to provide a frame of reference for creating or justifying university entrepreneurship units as a liaison mechanism for development of the extension function through one of its approximations: the business approach.

The article shows the approaches, models, mechanisms and strategies for universityenvironment interaction. It also reflects on entrepreneurship as a medium for that interaction; and provides an idea of the structural conditions that favor university entrepreneurship.
\end{abstract}

\section{Keywords}

Academic progress, education articulation, educational management, educational effectiveness (Source: UNESCO Thesaurus). 


\section{Interação universidade e ambiente: marco para o empreendimento}

\section{Resumo}

O documento desenvolve o tema da relação universidade-ambiente, especialmente no que se refere à vinculação com o setor produtivo como parte do desenvolvimento da função de extensão universitária.

O propósito fundamental é proporcionar um marco de referência para a criação ou a justificação das unidades de empreendimento universitárias como mecanismo de ligação para o desenvolvimento da função extensão a partir de uma de suas aproximações: o enfoque empresarial.

Como resultado, evidenciam-se os enfoques, os modelos, os mecanismos e as estratégias de interação universidade-ambiente; realiza-se uma reflexão sobre o empreendimento como meio para essa interação e mostram-se as condições estruturais que favorecem o empreendimento universitário.

\section{Palavras chave}

Progresso acadêmico, articulação educativa, gestão educativa, eficácia educativa (Fonte: Tesauro da Unesco). 


\section{Introducción}

Este documento de reflexión surge como avance de la investigación que responde al proyecto institucional denominado Integración UniversidadEmpresa-Estado, de la Universidad Militar Nueva Granada (UMNG), y muestra la necesidad de las universidades de fortalecer su vinculación con el entorno para dar respuesta a los requerimientos de la sociedad. Mediante el análisis efectuado, se pretende aportar en el marco de referencia para la creación de las unidades de emprendimiento de las organizaciones universitarias, en este caso específico, el marco general para la creación de la Unidad de Emprendimiento e Innovación de la UMNG, como una de las estrategias para el desarrollo de su función misional de extensión desde uno de sus enfoques: el empresarial. El documento presenta un marco general de los mecanismos y las estrategias de vinculación de la universidad con el entorno hasta centrarse en el emprendimiento. Se estructura de la siguiente forma: inicialmente, muestra los enfoques, modelos, mecanismos y estrategias de interacción universidad-entorno, seguido de una reflexión sobre el emprendimiento como mecanismo o estrategia de vinculación y las condiciones estructurales que favorecen el emprendimiento universitario.

\section{Enfoques, modelos, mecanismos y estrategias de interacción universidad-entorno}

La universidad, como organización que tiene entre sus actividades la generación, difusión y transferencia de conocimiento, se ha convertido en un actor fundamental en la nueva economía, que entiende el conocimiento como un factor estratégico que genera ventajas competitivas para permitir la diferenciación de las organizaciones y su sostenibilidad en el contexto. Conscientes de esto, las universidades han empezado a dar mayor importancia a una de sus funciones sustantivas: la extensión universitaria o proyección social, mediante la cual espe- ran llevar conocimiento al entorno para contribuir al desarrollo local, regional, nacional e internacional.

En este sentido, la educación debe "fomentar la construcción de conocimiento (docencia) y la investigación científica (investigación) que generen impactos positivos en la sociedad pero también debe permitir la identificación de oportunidades para el desarrollo regional o nacional y para el beneficio de la comunidad (extensión-proyección social)" (Ospina y Sanabria, 2010, p. 112).

\section{La extensión universitaria o proyección social como eje de articulación con el entorno y enfoques de interacción}

Aunque la universidad surge alrededor de las escuelas monacales (escolástica), en los siglos XII y XIII, teniendo como referentes principales "la de París (1215) en Francia, la de Bolonia en Italia y la de Oxford en Inglaterra" (Restrepo, 1999, p. 19), la concepción de universidad moderna surge alrededor de 1810 con la creación de la Universidad de Berlín en Alemania, reconociendo entre sus funciones sustantivas la docencia e investigación. En 1873, la Universidad de Cambridge, en Inglaterra, usó por primera vez el término extensión educativa, convirtiéndose en la tercera función sustantiva y en el elemento dinamizador que facilita el flujo continuo de conocimientos entre la universidad y la sociedad (Morales, Mira y Arias, 2010; Vega, 2002).

El propósito de la tercera función era la apertura de la universidad y su proyección social, tratando de responder a las necesidades de la comunidad. En estos inicios, las actividades de extensión se focalizaron en acercar la academia a la comunidad, a través de cursos de extensión en educación para adultos y capacitación para las comunidades circundantes, principalmente conferencias en agricultura (González y González, 2003; Torres, Sánchez y Márquez, 1996). En América Latina, se habla de la función social de la universidad a partir de 1918 cuando, en el 
manifiesto del Movimiento de Córdoba, uno de los principios estaba dirigido al fortalecimiento de la función social de la misma a través de la tercera función sustantiva - la extensión-, vinculando la "universidad al pueblo" con el propósito de poner el saber universitario al servicio de la sociedad y hacer de sus problemas tema fundamental de sus preocupaciones (Rofman y Vázquez, 2006).

Teniendo en cuenta que la universidad es una institución dinámica, Morales, Mira y Arias (2010) plantean que la función de extensión debe responder a diferentes contextos, haciendo frente a los procesos de globalización, democratización de la enseñanza y cambios políticos, para así estar en correspondencia con las demandas de la sociedad. En este sentido, dichos autores identifican tres enfoques de extensión en los que vienen trabajando las universidades:

- Uno de corte cultural, cuyo fin es la transformación permanente de la cultura — saberes, conocimiento, habilidades, aptitudes y valores, arte, tecnología, experiencias - (Torres, Sánchez y Márquez, 1996, p. 6). Este busca la participación de todos en la misma cultura universitaria acercando las comunidades. Esto implica que la universidad debe preservar, desarrollar y promover la cultura de la sociedad a través de la articulación de sus funciones sustantivas para corresponder a su cometido social (Vega, 2002).

- $\quad$ Otro de proyección social, que debe llevar a la academia, los estudiantes, los docentes y sus egresados a la búsqueda y solución de los problemas de la sociedad. Estos dos enfoques son propios de Latinoamérica y surgen como consecuencia de la Reforma de Córdoba que proponía conservar el carácter social de las instituciones universitarias (González y González, 2003; Torres, Sánchez y Márquez, 1996; Vega, 2002).

- El tercer enfoque está más vinculado con lo que se conoce como los conceptos universidadempresa, universidad-industria o universidad empresarial, los cuales han sido propios de los países en desarrollo de economía neoliberal y de las universidades de Europa Occidental y Estados Unidos que, sin alejarse de muchos de los compromisos sociales (economía, cultura y responsabilidad social), han estado comprometidas principalmente con el desarrollo industrial y la prestación de servicios que correspondan con los cambios del entorno. Así, las universidades han sido parte fundamental en el desarrollo a nivel regional, primordialmente a través de la transferencia de tecnología y la venta de servicios públicos y privados (González y González, 2003; Vega, 2002).

Según Ortiz y Morales (2011), aun cuando se puede evidenciar que las universidades latinoamericanas, y específicamente las colombianas, desarrollan actividades de extensión universitaria en los tres enfoques: empresarial, social o cultural, el grado de desarrollo de cada uno depende de factores del entorno regional y de los propósitos particulares de las diferentes instituciones universitarias. Sin embargo, es claro que el modelo económico dominante a nivel mundial ha hecho que la mayoría de universidades busquen organizar sus actividades de extensión de tal forma que puedan responder a las necesidades de su entorno local, regional y nacional, siendo evidente una tendencia hacia el desarrollo de la función de extensión desde el enfoque empresarial como un requisito del mercado y, específicamente, del sector productivo.

De acuerdo con lo anterior, se deja como reflexión que la universidad latinoamericana $-\mathrm{y}$, por supuesto, la colombiana- debe tener cuidado de no abandonar o dejar de lado una perspectiva amplia que incorpore los tres enfoques de extensión identificados, ya que no sería conveniente privilegiar solo el enfoque empresarial impuesto en los últimos años por las dinámicas del mercado y que es muy destacado en las universidades anglosajonas. Esto teniendo en cuenta que la región enfrenta 
unas problemáticas particulares que requieren del liderazgo de las universidades, no solo en la solución de problemas empresariales, sino en la atención a los problemas sociales y la conservación de su acervo cultural.

\section{Modelos de relación academia-entorno}

Se han planteado distintos modelos teóricos tendientes a explicar la integración de las universidades con otros actores de la sociedad (Pineda, Morales y Ortiz, 2011). Lo anterior, teniendo en cuenta que el conocimiento es producido por diversos actores, como las universidades, los centros de desarrollo tecnológico, las empresas, los laboratorios estatales y privados, entre otros, y que son tres vértices los que principalmente estructuran el campo de generación y circulación de conocimiento: los sistemas políticos, los sistemas económicos y los sistemas educativos (Maldonado, 2008).

De acuerdo con Pineda, Morales y Ortiz (2011), las vinculaciones entre la academia y el entorno han sido abordadas desde modelos como el triángulo de Sábato y Botana (1986), el de la triple hélice (Etzkowitz y Leydesdorff, 1995) y el de sistemas de innovación (Lundvall, 1997), entre otros. Estos modelos, especialmente el de la "triple hélice", le asignan un rol protagónico a la universidad en la generación y transferencia de conocimiento e innovación hacia la sociedad.

Alrededor de dichas vinculaciones se encuentran apreciaciones que sustentan que mientras en los países desarrollados las contribuciones se concentran en el fortalecimiento de los vínculos universidad-industria a partir de la realización de investigaciones conjuntas, el desarrollo de patentes y la transferencia de tecnología (Tornatzky, Waugaman y Gray, 2002), en los países emergentes, los principales aportes de la universidad se orientan hacia la formación del capital humano, la adaptación de las innovaciones tecnológicas y su difusión en las empresas locales (Schiller, 2006). De cualquier forma, lo que se evidencia es que, tanto en los países industrializados como en las economías emergentes, se están trabajando diversos mecanismos y estrategias que permitan llevar a cabo esta vinculación, aunque en su mayoría enfocados más a la relación de la universidad con el entorno empresarial (tercer enfoque de la extensión).

\section{Mecanismos y estrategias de vinculación universidad-entorno}

Existen diversos mecanismos y estrategias para la vinculación de la universidad con el entorno, los cuales pueden variar según el contexto de los países desarrollados y los países en desarrollo (Torres et al., 2009). En el caso de los países desarrollados, se presenta una tendencia creciente de las corporaciones privadas a crear alianzas estratégicas con universidades, y, en muchos de ellos, se están introduciendo leyes sobre propiedad intelectual para favorecer la interacción. Los principales mecanismos y estrategias son las spin offs, spin outs y start ups, ${ }^{2}$ las oficinas de transferencia de tecnología (OTRI), ${ }^{3}$ las incubadoras de empresas, los parques científicos y tecnológicos, ${ }^{4}$ las pasantías y prácticas profesionales, las consultorías, los programas de educación continuada, los centros de investigación cooperativa y de tecnología, las organizaciones re-

2 Cabe aclarar que las start ups son los emprendimientos de base científica y tecnológica jóvenes, surgidos de procesos de incubación, las spin off son iniciativas empresariales de base científica y tecnológica que se encuentran en una fase madura, mientras que las spin outs son iniciativas empresariales surgidas de la independencia de una sección o área de una empresa de base tecnológica (Etzkowitz, 2002).

3 Son organizaciones híbridas que se centran en identificar las necesidades tecnológicas de los sectores socioeconómicos y en favorecer la transferencia de tecnología entre el sector público y el privado, contribuyendo así a la aplicación y comercialización de los resultados de la I+D+l generada en las universidades y los centros públicos de investigación.

4 Los parques científicos y tecnológicos son estructuras que estimulan y gestionan el flujo de conocimiento y la tecnología entre universidades, instituciones de investigación, empresas y mercados; impulsan la creación y el crecimiento de empresas innovadoras mediante mecanismos de incubación y generación centrífuga (spin off), y proporcionan otros servicios de valor añadido, así como espacios e instalaciones de gran calidad (Morales, Plata y Casallas, 2011). 
gionales de innovación, las alianzas estratégicas o los consorcios, los centros o unidades empresariales y las políticas institucionales y nacionales, entre otros (Pineda, Morales y Ortiz, 2011).

En economías en desarrollo como las latinoamericanas, son muy recientes y reducidos los esfuerzos para el establecimiento de una infraestructura física y social para el fortalecimiento de estas interacciones; parte de ello se evidencia en los escasos proyectos de investigación realizados en alianzas entre el sector productivo y académico, en la falta de claridad sobre el manejo de propiedad intelectual y en patentes o niveles de emprendimiento bajos, que en su mayoría son por necesidad y no por oportunidad (Pineda y Morales, 2010; Pineda, Morales y Ortiz, 2011). Entre los mecanismos y las estrategias de interacción más comunes en la región están las pasantías o prácticas estudiantiles, además de los diplomados, las consultorías profesionales y las asesorías organizacionales brindadas por las universidades, a través de áreas administrativas con denominaciones cercanas a lo que podrían ser unidades de extensión. Más recientemente, se han promovido esfuerzos en el establecimiento de unidades de emprendimiento e incubadoras de empresas de base científica y tecnológica (Sutz, 2000).

En este campo, se destacan las universidades estadounidenses, que cuentan con amplias ventajas respecto de otras del mundo en la materialización de relaciones entre las universidades y el entorno, favorecidas por una alta financiación, la protección de la propiedad intelectual, la interdisciplinariedad en la investigación y la descentralización en la normatividad que regula las actividades científicas y tecnológicas.

El estudio de los mecanismos y las estrategias de relación entre las universidades y el entorno en diferentes contextos, ha señalado que las estrategias para relacionarse tienden a repetirse, indiferentemente de las necesidades propias de cada región, sin atender a que estas relaciones dependen tanto de las condiciones externas e internas de las instituciones, como de sus dimensiones gerencial, operacional y adaptativa, e incluso de las habilidades personales de quienes las conforman (Pineda, Morales y Ortiz, 2011; Torres et al., 2009). En este sentido, las universidades deberían inicialmente tener claras tanto sus capacidades en investigación y docencia (Sanabria, 2015) como las necesidades del entorno, para entrar a definir la orientación que debe tener la función de extensión o proyección social y los mecanismos o las estrategias que se requieren para articularse con el entorno.

\section{Retos para la universidad frente a la vinculación con el entorno}

Existe una gran cantidad de experiencias en las universidades latinoamericanas que muestran los diferentes caminos escogidos y de las que se pueden aprender valiosas lecciones. Es claro que los procesos de interacción universidad-sociedad deben ser el centro y la razón de ser de los programas de extensión. Por esto, la reflexión sobre esta función sustantiva debe ser un proceso permanente y debe tener un reconocimiento y unos responsables dentro de la estructura de las universidades que permitan una planeación y una ejecución coordinada con las otras dos funciones: la docencia y la investigación, lo que permitirá una mayor pertinencia del quehacer de las instituciones de educación superior en su contribución a la solución de las diversas problemáticas de sus entornos (Ortiz y Morales, 2011, p. 361).

Otros retos en relación con lo anterior son:

- Establecimiento de políticas y reglamentación de la extensión: la universidad debe establecer políticas y reglas de juego claras frente a la extensión (proyección social) de tal forma que sea evidente que está respondiendo a las necesidades de su contexto, sobre todo por su origen público y que, en el caso latinoamericano, se espera de ella no solo una respuesta hacia el sector productivo, sino también hacia 
la sociedad, desarrollando innovaciones sociales que involucren diversas instituciones y den solución a las problemáticas de la región (Morales, Pineda y Ávila, 2011). De igual forma, se requiere claridad sobre el manejo de la propiedad intelectual y la transferencia de conocimiento, de manera que estén acordes con la realidad que experimentan los investigadores colombianos y les permitan proteger y explotar sus desarrollos.

- Formación de capital humano competente: es necesario incentivar la formación permanente de docentes e investigadores en temas relacionados con la comercialización de ciencia y tecnología, capacitar facilitadores de vínculos con actores externos y estimular la formación en emprendimiento en los currículos académicos. Esto permitirá aumentar la acumulación de conocimiento e incentivar la producción intelectual requerida para la consolidación de una base científica que pueda ser llevada al entorno.

- Revisión y fortalecimiento de las pasantías universitarias: aunque existen beneficios para los actores involucrados, las críticas muestran que es necesario vincular el proceso de pasantías tanto a las dinámicas relativas al fortalecimiento de los planes de estudio de los diferentes programas como a los procesos de investigación aplicada que desarrollan los grupos de investigación, de manera que la universidad contribuya de forma efectiva a la solución de las problemáticas que se presentan en las diferentes organizaciones productivas, sociales y del Estado.

- Fortalecimiento de redes de apoyo: las redes de apoyo son un efectivo canal de comunicación entre los actores que es necesario consolidar si se desea que haya interacción dinámica entre los centros-grupos de investigación de la región (en las diferentes áreas del conocimiento) y las empresas, el Estado y los demás actores interesados. Establecer una infraestructura que favorezca estas interacciones requiere de la construcción de puentes entre el Gobierno, las universidades y las organizaciones de manera transdisciplinaria, para el aprovechamiento del capital humano y las capacidades científicas y tecnológicas existentes (Pineda y Morales, 2010).

- Generación de estructuras de soporte a la extensión: una acción fundamental para el adecuado desarrollo de la extensión es la generación de una cultura y estructura organizacionales que sustenten adecuadamente la función de extensión, a fin de evitar las limitaciones de enfoque que ya fueron señaladas.

- Creación de mecanismos para la gestión y transferencia de conocimiento: un desafio adicional es crear un parque tecnológico que mediante diversos mecanismos materialice la interacción con los diversos actores de la sociedad (Morales, Plata y Casallas, 2011), y donde se articule una unidad de emprendimiento que gestione y direccione proyectos de emprendimiento sostenibles que se soporten en el conocimiento y que puedan ser llevados a incubación. La incubación de organizaciones se presenta como una estrategia para el acompañamiento y la aceleración de emprendimientos de distinto tipo (Pineda y Morales, 2011), aunque es deseable que se incuben proyectos que ostenten algún nivel de innovación. De esta forma, se podría generar un clúster de conocimiento conformado por diversas organizaciones y spin offs 5 que apalanquen el desarrollo económico y social de la región y del país.

En este último marco, y a partir de la premisa de que las universidades se han interesado en fomentar la cultura del emprendimiento por tratarse

5 Entendidas como empresas de base científica y tecnológica constituidas desde las universidades. 
de una estrategia para transferir conocimiento al entorno, a continuación se presenta un marco referencial sobre el emprendimiento. Este marco será planteado desde las tres funciones misionales de la universidad, en razón a que este debe concebirse como una manera de pensar y actuar que debe partir desde la formación, articularse con la investigación y ser llevado al entorno a través de la extensión.

\section{El emprendimiento en las universidades}

En primera instancia, es importante reiterar que la función de extensión o proyección social no puede limitarse exclusivamente al desarrollo del emprendimiento o, aún peor, solo al emprendimiento empresarial, dadas las razones ya señaladas en el primer segmento. Así lo entienden algunas de las universidades más reconocidas en el país, en las que se considera al emprendimiento como uno más de los mecanismos de interrelación de la universidad con el entorno.

Sin embargo, en esta parte del documento se reivindica al emprendimiento como una de las modalidades o campos de acción de la función de extensión cuyo papel ha sido reconocido como de gran importancia para las universidades y para la sociedad (Alvarado y Rivera, 2011).

En este sentido, a continuación se establecerán las razones de esta relevancia y la justificación del emprendimiento a partir de las tres funciones sustantivas de la universidad. En el desarrollo de este tema se establecen los conceptos fundamentales del emprendimiento.

\section{La importancia del emprendimiento en la educación superior}

En los últimos años, se ha hecho evidente que las instituciones de educación superior (IES) han incorporado el tema del emprendimiento a sus procesos de formación, extensión e investigación, con diferentes énfasis, estrategias y acciones, debido a la importancia que se le ha dado en función del desarrollo económico y social. Los gobiernos también lo han vinculado dentro de sus agendas y han estructurado planes, programas y proyectos para fortalecerlo (Gibb y Hannon, 2007), entendiendo que su promoción puede contribuir al desarrollo económico y social de los países (Alvarado y Rivera, 2011; Cardona et al., 2008; Gámez, 2009; Rosa y Bowes, 1990, citados en Gómez y Satizábal, 2011).

En general, el fenómeno del emprendimiento puede entenderse como "la combinación entre la actitud y la capacidad de la persona, que le permiten llevar a cabo nuevos proyectos de cualquier índole, generalmente creativo. Se puede decir que el emprendimiento es la acción de convertir una idea (abstracta) en algo concreto (real)" (Morales, 2009). Por tanto, de forma amplia, el emprendimiento no necesariamente está relacionado con la creación de una organización o el beneficio económico; tal como lo señala Kunkel (1991, citado en Dehter, 2002), “El 'ser emprendedor' no está ligado inextricablemente a la idea de 'negocio lucrativo', ni a la 'innovación aplicada a la creación o mejora de bienes o servicios', ni al autoempleo o la satisfacción de necesidades económicas personales".

Lo anterior se esclarece completamente si se considera que el origen etimológico de la palabra emprendimiento "deriva del término francés entrepreneur, que significa estar listo a tomar decisiones o a iniciar algo" (Rodríguez, 2009), pero puede volverse difusa si se considera la traducción del término inglés entrepreneurship pues, dada su ambigüedad, puede llegar a ser traducida como emprendimiento, empresarialidad, espíritu emprendedor, empresariado o incluso la de emprendedurismo (Gutiérrez, 2011).

Sin embargo, una derivación concreta de esta noción puede definir el emprendimiento como el desarrollo de un proyecto que tiene un propósito económico, político o social, y que posee ciertas características de incertidumbre e innovación (Formichella, 2004). 
Al respecto, algunos autores hablan de diferentes modelos para comprender el emprendimiento. En el caso de Gámez (2009), se establecen al menos tres: 1) del evento emprendedor (Shapero y Sokol, 1982, citados en Gámez, 2009), que muestra las motivaciones que llevan a un emprendedor a tomar la decisión de crear empresa a partir del atractivo y viabilidad percibidos; 2 ) de los factores precipitantes (Veciana, 2001, citado en Gámez, 2009), que muestra que el emprendedor decide emprender por razones como la insatisfacción con la actividad actual, la identificación de una oportunidad de negocio o la visualización de una necesidad insatisfecha (aunque existan factores como la experiencia, trayectoria, historia - factores fundamentales- que lo facilitan); 3) The Global Entrepreneurship Monitor (GEM), que muestra las influencias de la sociedad y del entorno en el emprendimiento (las instituciones existentes, la competencia dada, los procesos de innovación, las condiciones personales del emprendedor, las oportunidades presentes y la dinámica empresarial vigente).

La inclusión de este concepto en la educación superior se ha visto justificada a partir de planteamientos como el de la Unesco (1998), que en su Declaración Mundial sobre la Educación Superior para el siglo XXI, afirma que el rol de la universidad debe contemplar la necesidad de llevar los conocimientos al mundo del trabajo, y preocuparse por las acciones para "aprender a emprender y fomentar el espíritu de iniciativa", con el fin de facilitar las condiciones de trabajo una vez concluida su formación profesional.

El reto para las universidades parece ser, entonces, la formación de individuos capaces de iniciar proyectos de distinta índole (económicos, políticos, públicos o sociales), de propiciar el cambio y el crecimiento de beneficios colectivos o, incluso, de asumir riesgos moderados y calculados como el que implica la creación de una empresa (Ecotec, 2007). La formación, por tanto, deberá fundamentarse en motivadores diferentes a la necesidad, la supervivencia económica o la generación de riqueza, con proyección siempre hacia el futuro (Fontela, Guzmán, Pérez y Santos, 2006), pues la capacitación para los negocios y para la creación y administración de empresas no es suficiente para el desarrollo de las competencias propias de un emprendedor (Kirby, 2004). Esto habrá de ser uno de los principales diferenciadores entre el emprendimiento comercial y el emprendimiento social (Guzmán y Trujillo, 2008).

Se consideran entonces fundamentales en la formación del emprendedor las siguientes habilidades: técnicas (comunicación oral y escrita, trabajo en red), de gestión empresarial (planeación y conocimientos técnicos) y personales (asumir riesgos, innovación y constancia) (Hisrich, Peters y Shepherd, 2005; Kirby, 2004; Sarasvathy, 2001).

En Colombia, el emprendimiento es entendido como:

... una manera de pensar y actuar orientada hacia la creación de riqueza; es una forma de pensar, razonar y actuar centrada en las oportunidades, planteada con visión global y llevada a cabo mediante un liderazgo equilibrado y la gestión de un riesgo calculado; su resultado es la creación de valor que beneficia a la empresa, la economía y la sociedad (Ley 1014 de 2006, 1).

No obstante, uno de los paradigmas dominantes sobre el que se sustenta el tema, y que es compartido por la gran mayoría de universidades, se refiere al desarrollo de la capacidad empresarial y la creación de empresas. En este sentido, el emprendimiento ha sido vinculado con riqueza, empresa, cambio, empleo, valor, crecimiento, pero posiblemente, la perspectiva más asociada a este ha sido la de innovación.

Esta visión del emprendimiento se relaciona con el establecimiento de una nueva función que bien puede ser la introducción de un producto novedoso o de un método diferente de producción, la apertura de mercados inexplorados, el contacto con nuevas fuentes para el suministro de materiales o 
la apertura de una organización en cualquier industria pero no necesariamente con la constitución de un proyecto que desborde las fronteras del conocimiento científico, técnico o tecnológico, lo cual podría ser altamente excluyente y presentaría una visión extremadamente limitada de este concepto que, en realidad, se sabe sustentado en una ideología para la inclusión (Ararat, 2010).

Por su parte, Toca (2010) sostiene que el equivalente en castellano del término emprendimiento está asociado a la innovación, al inicio de proyectos propios, a la apertura de organizaciones en cualquier área o, en términos concretos, al proceso de creación de valor. Las oportunidades constituyen el núcleo del emprendimiento, por lo que emprender implica vincular oportunidades con recursos disponibles e identificar claramente aquellas fuentes de las que se pueden obtener los recursos demandados. Esta noción de innovación ha estado vinculada con el emprendimiento desde el origen mismo del concepto, pues desde entonces se planteaba que el emprendedor tenía como función reformar los modos de producción, generar invención, crear un nuevo producto o nuevas maneras de fabricarlos, etc. (Schumpeter, 2003, p. 132).

En el mismo sentido, la Organización para la Cooperación y el Desarrollo Económicos (OCDE) considera como innovación "la introducción de un nuevo, o significativamente mejorado, producto (bien o servicio), de un proceso, de un nuevo método de comercialización o de un nuevo método organizativo, en las prácticas internas de la empresa, la organización del lugar de trabajo o las relaciones exteriores" (OCDE y Eurostat, 2006, p. 56).

En consecuencia, dado que existe una marcada relación entre los conceptos de emprendimiento e innovación, pero que también "es posible tener emprendimiento sin innovación [...] e innovación sin creación de empresas nuevas" (Vesga, s. f.), es posible establecer la existencia de lo que podría denominarse emprendimiento innovador, es decir, un emprendimiento que incluya una manera de pensar y actuar orientada a asumir riesgos económicos y sociales, para crear nuevas organizaciones o fortalecerlas, que generen valor a través de la explotación de nuevas ideas en el desarrollo de productos, procesos o métodos nuevos o significativamente mejorados. Así, su resultado contribuye al desarrollo económico y social (OCDE y Eurostat, 2006) o involucra "el proceso deliberado que permita el desarrollo de nuevos productos, servicios y modelos de negocios en las organizaciones" (Vesga, s. f.).

Estos emprendimientos innovadores pueden incluir tanto Investigación y Desarrollo (I+D), como también "numerosos trabajos que no incluyan I+D pero que formen parte de la innovación. Estas actividades pueden reforzar las capacidades que permiten desarrollar innovaciones o la aptitud para adoptar con éxito innovaciones desarrolladas por otras empresas o instituciones" (OCDE y Eurostat, 2006, p. 45).

En este sentido, la innovación puede incluir: a) la definición de nuevos conceptos de producto, proceso, métodos de comercialización o cambios organizativos; b) la compra de información técnica, pago de derechos por invenciones patentadas o compra de conocimientos tecnológicos y experiencia; c) el aumento de la experiencia profesional o el aprendizaje táctico e informal; d) la inversión en equipos, programas o insumos intermedios que incorporen la innovación realizada por otros; e) la reorganización de los sistemas de gestión y el conjunto de las actividades empresariales, o f) el desarrollo de nuevos métodos de comercialización y venta de bienes y servicios (OCDE y Eurostat, p. 2006).

Se aprecia, entonces, que el emprendimiento es muchas cosas a la vez: proceso, actividad, conducta y habilidad. Es un proceso que permite descubrir una manifestación o revelación de ineficacias temporales y espaciales de una economía. Es una actividad que contempla el descubrimiento, la valoración y la exploración de oportunidades para introducir nuevos bienes y servicios, y modos de organizar mer- 
cados y procesos, y que puede ser definida como "la gestión del cambio radical y discontinuo, o renovación estratégica, sin importar si esta ocurre dentro o fuera de las organizaciones existentes, y sin importar si la renovación da lugar, o no, a la creación de una nueva entidad de negocio" (Kunkel, 1991, citado en Dehter, 2002, p. 19).

Por otra parte, como lo menciona Formichella (2004), el emprendimiento no solo es de carácter económico, sino que puede ser también de carácter político o social. En este sentido:

Todo emprendimiento social incluye tres elementos clave: un objetivo social, una innovación transformadora y un modelo de negocios sostenible. El emprendimiento social suele darse, y tener éxito, en los sectores de la economía donde el mercado ha fallado y la acción del Estado es inexistente o ineficaz. Un emprendimiento social, cuando resulta exitoso, puede no solo transformar las vidas de los beneficiarios, sino también la forma de abordar un problema en general (Ramírez, 2008).

Toca (2010) afirma que dado que la habilidad para el emprendimiento no se encuentra igualmente desarrollada en todos los individuos, y que por ello debe enseñarse, esta capacidad puede ser efectivamente desarrollada y enseñada (Gibbs, 2005, citado en Gámez y Satizábal, 2011). Su enseñanza - de preferencia holista - exige un compromiso multisectorial en ambientes diversos, cambiantes e inciertos. Por ello, los países han construido, como en el caso de Colombia, instituciones para promover el emprendimiento con educación (Gámez, 2009).

Por ello es que para Kantis et al. (2001), hay un marcado interés en el estudio de la relación entre educación universitaria y emprendimiento, a partir de la necesidad de impulsar el surgimiento de nuevas empresas basadas en el conocimiento y la tecnología, que ha generado la implementación de programas e iniciativas orientadas a desarrollar el potencial que existe en las universidades para la creación de este tipo de empresas.
Bajo esta misma concepción, Etzkowitz (2004) señala que el espíritu empresarial académico es una extensión de las actividades de enseñanza y de investigación, y una manera de interiorizar sus capacidades de transferir tecnología y capitalizar el conocimiento cumpliendo un rol como actor económico por derecho propio y como generador de procesos de innovación.

Por ello es que este autor considera que:

El importante rol de las nuevas empresas creadas por egresados universitarios [...] es especialmente crucial en los paises emergentes como Argentina (y América Latina en general) ya que la estructura industrial está compuesta por firmas de sectores tradicionales caracterizados por un relativamente bajo o moderado contenido tecnológico y que no juegan un rol importante como "organizaciones incubadoras" de nuevos emprendedores dinámicos (Kantis et al., 2001, p. 4).

El autor realiza un estudio en Argentina para identificar las diferencias existentes entre emprendedores universitarios y aquellos que no lo son. Encuentra que las empresas creadas por emprendedores de base universitaria se caracterizan por tener un perfil más calificado en cuanto a su mayor dinamismo, el talento humano que vinculan y el tipo de oportunidades que aprovechan, y porque aportan a la diversificación y el enriquecimiento de la industria; ello justifica la necesidad de promoverlas.

En este mismo sentido, Löbler (2006, citado por Toca, 2010) afirma que en ambientes diversos, cambiantes e inciertos, los individuos deben formarse en emprendimiento, aprender a tomar buenas decisiones y a conducir iniciativas o proyectos, a generar sus propias respuestas frente a los acontecimientos del entorno, a dejar de buscar soluciones en experiencias pasadas y a aprender más sobre el saber que sobre el hacer. 
Es así que la educación en emprendimiento se configura como una opción, una alternativa distinta, entre otras cosas, para la búsqueda de empleo, en donde los profesionales puedan crear su propio espacio de trabajo, generar riqueza, empleo e innovación, y así contribuir al desarrollo económico y social de los países. Esto se da al punto de configurar en el discurso incluso dos grupos sociales: los emprendedores y los empleados (Ararat, 2010). En este sentido, esta opción de educación debe responsabilizarse de formar al emprendedor y al intraemprendedor como se señala a continuación.

En cuanto a la formación del emprendedor, este tipo de educación debe formar una persona con capacidad de innovar - entendida esta como la capacidad de generar bienes y servicios de una forma creativa, metódica, ética, responsable y efectiva (Ley 1014 de 2006)—; de crear empresa, organizarla y gestionarla con iniciativa y asumiendo los riesgos inherentes a ello; de tener visión de negocio, compromiso, motivación y pasión para orientar a un grupo de personas -empleados-y convencer a inversionistas, proveedores y clientes; de aprovechar las oportunidades que detecta en el mercado, usar los resultados de sus investigaciones para su desarrollo, generar ventajas competitivas y poner en marcha los proyectos.

Por otro lado, en cuanto se refiere a la formación del intraemprendedor, esta opción de educación debe formar una persona que posea la visión, la pasión y la perseverancia de un emprendedor pero que, estando presente en el interior de las organizaciones, sea capaz de desarrollar innovaciones que las beneficien directamente. En este sentido, debe formar una persona con una aguda capacidad para identificar nuevas oportunidades de negocio que repercutan en la actividad de la organización para la que trabaja. "El emprendedor dentro de la empresa, asume la responsabilidad activa de producir cualquier tipo de innovación en la organización" (Pinchot, 2003).

\section{Desarrollo del emprendimiento a partir de las tres funciones sustantivas}

Si se observa cómo se integra el emprendimiento en las tres funciones de la universidad, se encuentra un énfasis en lo que compete a la formación: en la mayoría de instituciones se implementan y desarrollan programas, cursos y talleres de apoyo a los emprendedores, en algunos casos incorporados a la malla curricular, en otros, abiertos a los estudiantes que están interesados en el tema. Algunos críticos, sin embargo, advierten que en muchas instituciones los programas o cursos para emprendedores están más orientados a la elaboración de planes de negocio y seminarios sobre finanzas, marketing, marcos legales o descripciones del emprendedor, y que dejan de lado el desarrollo del capital social, es decir, los recursos individuales y las competencias específicas que permiten a las personas interactuar más efectivamente con otras. Estas cobran gran importancia para los emprendedores en aspectos como la selección de socios, la conformación de equipos y alianzas, el contacto con clientes e inversionistas, etc.

\section{Desarrollo en la docencia}

En una dirección similar, Gibb (2007, citado por Ortiz y Herrera, 2010) afirma que la educación en emprendimiento debe formar para soportar la ambigüedad y permitir los errores como posibilidades de aprendizaje y la formación de redes que sustenten todo el proceso emprendedor. Por tanto, es necesario reflexionar sobre si los espacios de una cátedra, en términos de número de horas semestrales, obligatoriedad de la evaluación numérica y criterios de homogeneización, permiten una adecuada formación para los potenciales emprendedores, o se limita a brindar unos conocimientos formales que llevan al estudiante a una situación pasiva frente a su propio proceso de aprendizaje. Es necesario tener siempre presente que el emprendimiento es el resultado de la interacción de múltiples factores internos y externos, que se concatenan 
de forma dinámica, en aras de la identificación de nuevas oportunidades.

De acuerdo con la literatura (Henrique y $\mathrm{Da}$ Cunha, 2008, citados por Toca, 2010), los programas que pretendan la enseñanza del emprendimiento deberían incluir en sus asignaturas habilidades de comunicación (persuasión), de creación, de reconocimiento de oportunidades emprendedoras, de liderazgo, de negociación, de toma de decisiones, de solución de problemas, de vinculación con redes y de administración del tiempo; asimismo, competencias gerenciales (planeación, comercialización, contabilidad, estrategia, marketing, gestión humana) y pensamiento crítico.

En el documento de Ortiz y Herrera (2010) se recoge el trabajo realizado en el año 2008 bajo la coordinación de la Asociación Colombiana de Facultades de Administración (Ascolfa) , titulado "Estado del arte del emprendimiento en las facultades de administración en Bogotá: investigación, promoción y formación en el periodo 2002-2007", cuyo propósito fue explorar las acciones realizadas por las universidades en torno al tema del emprendimiento.

En total, de las universidades estudiadas, el $81 \%$ incluyen el tema de alguna forma en sus documentos institucionales. Esto permite pensar que, desde hace algunos años, en la formación se contempla la necesidad de desarrollar competencias o de ofrecer posibilidades profesionales diferentes a las tradicionalmente contempladas y ello ha permitido la vinculación del emprendimiento. El estudio también permite identificar que son mayoritariamente las facultades de administración o de ciencias económicas las que se han responsabilizado del tema, seguidas de la conformación de centros específicos que desarrollan actividades para toda la universidad ya que, en general, se considera una competencia transversal para todos los profesionales.

Como se mencionó, el estudio evidencia que la formación en emprendimiento se aborda en el 95\% de las facultades de administración en Bogotá, esto permite ver claramente que es una temática que ya se insertó dentro de los currículos y hace parte de los procesos de formación, aunque con diferencias en cuanto a la intensidad, la profundidad y las metodologías trabajadas.

Los resultados muestran que tanto a nivel de pregrado como de posgrado, el mayor porcentaje de actividad en el desarrollo de la cultura de emprendimiento se realiza bajo la modalidad de cátedra. En el pregrado se ofrecen cátedras en el 93\% de la población encuestada. También se evidencia que el tema ha penetrado mucho menos en los posgrados ofrecidos por las instituciones estudiadas: solamente el $27 \%$ de ellas reportan la inclusión de las cátedras dentro de estos programas. Estos datos permiten ilustrar la forma en que las IES, en nuestro contexto, han abordado el tema del emprendimiento y cómo han organizado sus esfuerzos para trabajarlo en lo que se refiere a la primera de sus funciones sustantivas: la formación.

\section{Desarrollo en la investigación}

La investigación en el tema de emprendimiento también presenta diversos desarrollos y énfasis de acuerdo con los intereses particulares de los investigadores del fenómeno, con los avances de los diversos grupos de investigación que se han conformado en torno al tema o con la importancia que le han otorgado las diferentes instituciones al emprendimiento.

En los diferentes eventos académicos, tanto en Colombia como en Latinoamérica, se puede evidenciar que el tema se constituye como un nuevo eje temático en torno al cual se reúnen un buen número de investigadores con diversas propuestas, metodologías y resultados que pretenden ampliar el cuerpo de conocimientos que existe sobre el emprendimiento. De esta forma, "el emprendimiento como temática de estudio de las ciencias económicas y de la gestión fue creciendo hasta configurar, si no un campo de investigación, al menos un interés emergente entre los investigadores que tratan de 
precisar las características de este fenómeno y en especial de analizar su estatus epistemológico" (Alvarado y Rivera, 2011, p. 63).

En tal sentido, aunque en el país no hay una base de datos que agrupe los estudios e investigaciones que se han llevado a cabo, en la plataforma Scienti de Colciencias se puede identificar que los grupos interesados en el tema han aumentado en la última década y se han desarrollado en instituciones de diferentes partes del país. En la actualidad, se pueden identificar nueve grupos visibles aunque es posible encontrar estudios referentes al tema en otros grupos.

Al realizar una agrupación preliminar de los estudios llevados a cabo por los diferentes grupos de investigación, se pueden encontrar las siguientes categorías:

Formación: proyectos relacionados con los procesos de formación (cátedras y otras actividades) que adelantan las instituciones educativas. Se mide también con alguna frecuencia la actividad emprendedora de los egresados de cada universidad.

Incubación: proyectos relacionados con creaciones de centros o entes encargados de apoyar la creación de empresas.

Pymes: proyectos que abordan diversas problemáticas de la pequeña y la mediana empresa desde la perspectiva del emprendimiento.

Empresarios: proyectos realizados teniendo como eje la historia empresarial colombiana o perfiles empresariales actuales.

Oportunidades: proyectos relacionados con la identificación de ideas de negocio.

Sectores: estudios que dan cuenta de la problemática específica de empresas y empresarios en algunos sectores económicos.

Finanzas: proyectos centrados en aspectos financieros de las empresas o los emprendedores.
Esta clasificación, muy general, permite identificar las tendencias y los intereses de los académicos que desean contribuir a generar conocimientos sobre el tema del emprendimiento.

\section{Desarrollo en la extensión}

Respecto a la función de extensión, el emprendimiento se liga con la visión actual de que la universidad es un agente de transferencia de conocimiento y de tecnología, y de que la base de este proceso son la innovación y el emprendimiento, teniendo siempre como fin último el compromiso social con el entorno en el que actúa e influye.

Bueno, Casani y Lizcano (1999), plantean que esta visión de la extensión se enmarca en el conjunto de actividades que las universidades llevan a cabo con los diferentes agentes sociales con los que se relacionan y a los que transfieren su conocimiento; en la transferencia de I+D que, en suma, genera la innovación que la sociedad reclama; en los esfuerzos que responden al compromiso social que tienen con su entorno como servicio público y en la generación de ingresos adicionales, aunque este no sea el objetivo último. Estos ingresos se derivan del proceso de comercialización tecnológica de los recursos universitarios.

Plantean los autores, que esto responde a la visión proactiva de la universidad para llevar a cabo acciones de desarrollo tecnológico, asistencia técnica, programas de formación continua, programas de posgrado y contratos de investigación, entre otras actividades, con corporaciones, fundaciones, empresas, entidades gubernamentales, asociaciones, etc. Se propone, entonces, una "tercera misión" de la universidad, a través de su actividad emprendedora, es decir, una misión construida visualizando una universidad para la transferencia de I+D o del conocimiento tecno-científico.

Esta comercialización tecnológica y esta función emprendedora se suele concretar en las nuevas políticas para facilitar y movilizar los procesos de 
creación de empresas de base tecnológica o spin offs universitarias y en la adecuada gestión de las patentes, modelos de utilidad y licencias, que se generan en una nueva relación entre la universidad y la sociedad a través, sobre todo, de las empresas y organizaciones que la integran (Bueno, Casani y Lizcano, 1999, p. 46).

Se propone, entonces, trabajar sobre dos ejes: la transferencia del conocimiento y el emprendimiento. El primero para que, en colaboración con los otros agentes del sistema de Ciencia y Tecnología, puedan concretarse innovaciones para el crecimiento y desarrollo sostenible de su entorno generando una visión de la universidad como espacio y agente de innovación. El segundo, que parte de esa transferencia de conocimiento tecno-científico a la sociedad, para que, como creadora de valor, riqueza y empleo, permita la comercialización tecnológica, la creación de spin offs académicas (producto de innovación) y la generación de nuevas relaciones entre los agentes.

\section{Condiciones estructurales que favorecen el emprendimiento universitario}

Como ocurre con la mayoría de aspectos de la realidad, el emprendimiento no se genera de forma espontánea y aislada, sino que se origina y depende de otros aspectos. Por tanto, para la comprensión y potenciación del emprendimiento se requiere de un conocimiento de los demás factores que lo afectan, es decir, la comprensión de aquellos aspectos que constituyen lo que se puede denominar la estructura para el emprendimiento.

En este apartado se van a evidenciar las variables que son consideradas como de mayor importancia para el fenómeno del emprendimiento y que, por supuesto, deberían ser tenidas en cuenta en el desarrollo de cualquier iniciativa de generación o fortalecimiento de este tipo de programas, ya sea en el entorno universitario, en el ámbito normativo o en el contexto organizacional.
En cuanto a la estructura requerida para el emprendimiento, es claro que en ella intervienen tanto factores económicos como aspectos sociales, culturales, políticos, ambientales, tecnológicos, etc. Por tanto, sin una adecuada estructura para el emprendimiento, cualquier esfuerzo que se realice para fomentarlo se constituirá en una acción aislada y con poca probabilidad de éxito.

Así, el emprendimiento requiere de la construcción de un ambiente propicio, que se encuentre más allá de las buenas intenciones y los propósitos gubernamentales, organizacionales o individuales, y de fines coyunturales, y que lleva a transitar hacia la intervención de los verdaderos factores que pueden limitar o impedir su adecuado desarrollo (aspectos estructurales). Kunkel (1991, citado en Dehter, 2002) considera que estos factores intervinientes pueden ser determinados por tres actores fundamentales: el Estado, las instituciones de educación superior y las empresas.

El Estado se considera como un actor importante para favorecer el emprendimiento al intervenir en variables como la generación de políticas, la creación de oportunidades, la reducción de impuestos, la desregulación del mercado, la determinación de sectores estratégicos, el apoyo a organismos de investigación y su vinculación con las organizaciones, la protección de las nuevas empresas y su incubación, y el incentivo a los demás actores para apoyar y participar en el emprendimiento.

Por otro lado, las instituciones de educación superior se consideran importantes porque favorecen el emprendimiento al afectar factores como: la formación para el emprendimiento y no solo para el empleo, la asistencia técnica que enriquezca la vocación emprendedora, el trascender los cursos de formación administrativa y autoayuda, el facilitar el ajuste entre los intereses y las vocaciones de los participantes y las oportunidades y los recursos existentes, y la verificación de la compatibilidad de las iniciativas con los intereses de la comunidad. 
En cuanto a las empresas, las variables sobre las que pueden intervenir para facilitar el desarrollo del emprendimiento son: fomentar el intraemprendimiento, la eliminación de los esquemas jerárquicos, el favorecimiento del comportamiento emprendedor, la creación de cultura de emprendimiento, la transmisión del emprendimiento a las asociaciones empresariales y profesionales, la promoción de las fortalezas de los intraemprendedores y la generación de sistemas de incentivos para retener a la gente creativa.

Por su parte, a partir de varios autores, Vesga (s. f.) ha planteado la existencia de tres niveles interactuantes que determinan el desarrollo del emprendimiento innovador:

En el nivel macro establece los componentes del entorno que afectan favorablemente a las organizaciones e individuos para emprender, a saber: a) mayor grado de producto per cápita, b) mayor tamaño del mercado, c) libertad para competir y mayores garantías para las empresas pequeñas, d) el uso de tecnologías y e) fluctuaciones favorables en las variables demográficas.

En el nivel de las empresas, este autor establece como variables que favorecen el emprendimiento las siguientes: a) la determinación de la estrategia y el compromiso con ella, b) el aseguramiento de los recursos y las capacidades para generar ventajas sostenibles, c) una cultura organizacional abierta, con jerarquías planas y multidisciplinares, y compromiso de la dirección con la innovación, y d) decisiones alineadas con los intereses de largo plazo de la organización. Paralelamente, en este nivel están las universidades que pueden favorecer el emprendimiento en: a) la producción del conocimiento, b) la difusión del conocimiento y c) la aplicación del conocimiento.

En el tercer nivel, el de los individuos, el emprendimiento se favorece a través de los siguientes aspectos: a) el alto nivel educativo, b) el desarrollo de habilidades venidas de la experiencia, c) el desa- rrollo de la capacidad de identificar oportunidades y atender necesidades, d) la disposición para el riesgo con la promesa de ganancias y e) la existencia de las cualidades o los recursos psicológicos requeridos (creatividad, perseverancia, iniciativa, autonomía, motivación, entre otras).

Este autor establece que las variables que pueden intervenirse para afectar las variables ya mencionadas son de dos tipos: las que conectan el nivel macro y el empresarial (instituciones, políticas gubernamentales y reglas de juego; infraestructura del país; acceso a capital para crecimiento; conocimiento sobre operación de la innovación y pertenencia a redes de este tipo; efectividad de la relación universidad-empresa) y variables que conectan el nivel macro y el de los individuos (instituciones y reglas de juego para la creación de empresas, acceso a capital para creación de empresas, incentivos para el comportamiento innovador, reducción del riesgo mediante la disponibilidad de información sobre oportunidades y pertenencia a redes que ayuden a superar las barreras).

De forma más específica, en el nivel del emprendimiento en el interior de las organizaciones (intraemprendimiento), otros autores han establecido como variables que afectan el emprendimiento a la estructura industrial, la competencia, el volumen de la oferta, el incremento de la demanda, el componente tecnológico, la ampliación de canales de distribución y la identificación de oportunidades y cambios del medio (Briceño, 2009). Asimismo, se mencionan los sistemas de incentivos, el soporte de la gerencia, el acceso a recursos, la estructura organizacional y el proceso de toma de riesgos como los factores que permiten el emprendimiento de este tipo (Christensen, 2005, citado en Briceño, 2009).

En la misma dirección, como resultado de su estudio sobre innovación, Briceño (2009) parece señalar que las variables más representativas para el favorecimiento del intraemprendimiento innovador son el respaldo tecnológico, el respaldo de los 
productos, la flexibilidad en la supervisión de los empleados, la posibilidad de planear de forma ascendente, el trabajo por resultados, los programas de incentivos y remuneración, el mayor tamaño de las organizaciones, la estructura organizacional horizontal, la comunicación abierta y los escenarios de discusión, los sistemas de información para conservar el conocimiento, la integración del proyecto de vida individual con la estrategia organizacional, la creación de políticas de formación, el aprendizaje de experiencias previas, la construcción de comunidades (clusters o distritos industriales) y la integración de las competencias individuales y colectivas al trabajo participativo.

En el estudio realizado por Morales (2009) con el fin de establecer la intención emprendedora de los estudiantes en las escuelas de negocios, y siguiendo con el análisis de los tres niveles inicialmente planteados por Vesga, parecen ser determinantes del emprendimiento las habilidades emprendedoras, el comportamiento y la intención ${ }^{6}$ del individuo (nivel de los individuos); la malla curricular de los programas, el enfoque de la enseñanza, la actitud de los profesores hacia el emprendimiento, los concursos de emprendimiento, las prácticas en microempresas y organismos sociales, las asesorías en la formulación y el desarrollo de proyectos, el financiamiento de proyectos, la existencia de profesores especialistas en el tema, las áreas internas de apoyo al emprendimiento -incubadoras, centros de emprendimiento, laboratorios-, las charlas sobre experiencias emprendedoras y los talleres de emprendimiento, entre otras (nivel de empresasuniversidades); las variables demográficas que afectan el comportamiento, el clima de emprendimiento en que se desenvuelve el individuo, la educación

$6 \quad$ La intención según Morales (2009), citando a Ajzen (1991), se explica por tres factores: la actitud hacia el comportamiento (evaluación personal), las normas subjetivas (presión social) y el control de comportamiento percibido (capacidad para realizar el comportamiento). formal y la educación orientada al autoempleo (nivel marco).

De otro lado, con la pretensión de evidenciar las limitaciones a la creación y el desarrollo empresarial, y citando varias fuentes, Giraldo, Bedoya y Vargas (2009) señalan como aspectos relevantes para el desarrollo emprendedor, en dos niveles, los siguientes:

En el primer nivel, el empresarial: 1) existencia y pertinencia del plan de empresa; 2) ventajas competitivas y comparativas existentes; 3 ) nivel de innovación tecnológica; 4) tamaño de las empresas (logro de economías de escala y sensibilidad frente al entorno); 5) condiciones técnicas y competitivas; 6) identificación y acceso a tecnología adecuada; 7) nivel de asociatividad empresarial; 8) capacidad gerencial y pensamiento estratégico de los directivos; 9) articulación del sector con la gran empresa y con los sistemas de compras estatales; 10) nivel de informalidad; 11) amplitud de los mercados donde se comercializa; 12) nivel económico y de formación del recurso humano; 13) acceso al sector financiero; 14) capacidad de innovación; 15) uso de tecnologías de información y comunicaciones; 16) posibilidad de comercialización de los productos; 17) acceso a insumos; 18) participación en el mercado de contratación pública; 19) capacidad de inversión; 20) origen de la iniciativa (necesidad u oportunidad).

En el segundo nivel, el macro: 1) crecimiento y desarrollo económicos; 2) legislación sobre la informalidad;3) infraestructura para investigación y desarrollo; 4) existencia de políticas estructurales que garanticen la permanencia de los nuevos empresarios.

De forma particular, en un análisis realizado desde una perspectiva institucional, Álvarez y Urbano (2011) señalan a las instituciones informales como las determinantes del emprendimiento en Latinoamérica, mientras las instituciones formales no juegan un papel significativo en el mismo. De esta manera, aspectos como la estabilidad política, el control de la corrupción y los modelos de referen- 
cia son los que influyen realmente en la actividad emprendedora (menores tasas de actividad emprendedora pero mayor calidad y sostenibilidad de las iniciativas), mientras factores como los procedimientos para la creación de empresas, el acceso a crédito bancario, la educación formal y las habilidades emprendedoras no parecen tener una relación significativa.

Por otra parte, dentro la reglamentación establecida en Colombia para el fomento del emprendimiento (Ley 1014 de 2006) se han considerado implícitamente algunos factores sobre los que se debería intervenir para favorecer su desarrollo y que pueden ser clasificados en cuatro categorías:

En la primera categoría, aspectos educativos: 1) promoción del espíritu emprendedor en el sistema educativo; 2) integración del sistema educativo y el sistema productivo; 3) formación de competencias para el emprendimiento en los programas académicos; 4) evaluación de la calidad de los programas de fomento al emprendimiento en el sistema educativo; 5) generación de un esquema de formación de formadores en el tema de emprendimiento; 6) creación de procesos de capacitación empresarial; 7) incorporación del emprendimiento a los currículos (preescolar a educación media); 8) generación de eventos de emprendimiento en el sistema educativo; 9) creación de la opción de grado universitario mediante un plan de negocio.

En la segunda categoría, aspectos culturales: 1) unificar criterios, conceptos y discursos sobre el emprendimiento en el país; 2) desarrollo de la cultura empresarial; 3) propender por la creación de comunidades y redes empresariales (cadenas y clusters productivos); 4) fortalecimiento del trabajo asociativo; 5) generar credibilidad en los planes de negocio que se desarrollen; 6) fomentar la cultura de cooperación, ahorro y asociatividad.

En la tercera categoría, aspectos económicos: 1) facilitar el acceso a capital (público) para las re- des de emprendimiento, 2) facilitar el acceso a crédito bancario para las nuevas empresas; 3 ) facilitar el acceso de las nuevas empresas a los inversionistas ángeles, fondos de capital semilla y de riesgo; 4) estandarización de la información y requisitos solicitados para acceder a recursos gubernamentales de cofinanciación; 5) generación de esquemas de cofinanciación como mecanismo de apoyo a las unidades de emprendimiento, entidades de apoyo a la creación de empresas, fundaciones, cámaras de comercio, universidades, incubadoras y ONG.

En la cuarta categoría, aspectos políticos y legales: 1) fomento y difusión del emprendimiento a través de la televisión pública; 2) reglamentación del emprendimiento; 3) creación de un sistema público que soporte el emprendimiento; 4) facilitar la creación y operación de las empresas; 5) reducción de trámites y costos para la formalización de nuevas empresas; 6) generación de servicios de orientación para la formalización empresarial; 7) formación y consultoría para emprendedores a través de las cámaras de comercio e incubadoras; 8) creación de medios para la comercialización de productos y servicios de emprendedores a través de las cámaras de comercio; 9) protección, incentivo y acompañamiento a las microempresas y pequeñas empresas; 10) creación de una red nacional para el emprendimiento; 11) creación de redes regionales para el emprendimiento; 12) facilitar el surgimiento de inversionistas ángeles, fondos de capital semilla y de riesgo; 13) articulación de los esfuerzos de los actores que promueven el emprendimiento; 14) formulación del plan estratégico nacional para el desarrollo de la cultura de emprendimiento; 15) creación de un sistema de seguimiento y apoyo empresarial para el emprendimiento; 16) facilitar la divulgación de los servicios existentes de apoyo al emprendimiento y ordenar la oferta existente; 17) mantener el emprendimiento como tema central en los planes, programas y proyectos de desarrollo; 18) organización de eventos de emprendimiento; 19) mantener actualizada la información sobre el desarrollo del 
emprendimiento en el país; 20) creación de concursos para el emprendimiento social y de negocios.

Como se puede evidenciar, la normatividad existente comparte algunos de los elementos tratados por los demás autores y que han sido considerados como relevantes para favorecer el emprendimiento en el país; sin embargo, también se puede verificar que la ley no alcanza a tener en cuenta muchas de las variables que se consideran como fundamentales para el desarrollo del emprendimiento. A pesar de ello, la cuestión esencial puede estar más bien en determinar la forma en que se puede intervenir efectivamente en ellas para ponerlas en acción, de forma que generen un impacto real en el desarrollo del mismo, pues a ocho años de la expedición de la ley parecen existir muchas tareas pendientes.

Por otro lado, aparte de las cámaras de comercio, el SENA, las fundaciones y las incubadoras de empresas, en este esquema planteado las unidades de emprendimiento toman una importancia fundamental al entenderse que estas son el "centro o programa de atención en donde se capacita y asesora a emprendedores durante los procesos de creación y consolidación de empresas. Estos centros o programas son ambientes de aprendizaje encargados de fomentar el desarrollo de la cultura del emprendimiento" (Ministerio de Comercio, Industria y Turismo, 2009).

En suma, dadas las diferentes aproximaciones referidas, y siguiendo la clasificación propuesta por Vesga (s. f.), parece existir una determinada estructura del emprendimiento que cuenta con variables identificables y bien diferenciadas que se pueden considerar como esenciales para el emprendimiento y que, por tanto, se deberían considerar como el referente de cualquier intervención para la generación y el desarrollo de este.

\section{Referencias}

Ajzen, I. (1991). The theory of planned behavior. Organizational Behavior and Human Decision Processes, 50, 179-211.

Alvarado, O. y Rivera, W. F. (2011). Universidad y emprendimiento, aportes para la formación de profesionales emprendedores. Cuadernos de Administración (45), 61-74.

Álvarez C. y Urbano D. (2011). Environmental factors and entrepreneurial activity in Latin America. Revista Latinoamericana de Administración-Cladea, 48, 31-45.

Ararat, J. (2010). La ideología del emprendimiento. Una mirada desde el análisis crítico del discurso. Revista Administer, 17, 5-33.

Briceño, M. (2009). La innovación como accionante del emprendimiento en las organizaciones. Borradores de Administración, 31, 1-21.

Bueno, E., Casani, F.y Lizcano, J. (1999). Formación de la estrategia empresarial: un análisis de las dinámicas del proceso estrategia. Revista Española de Financiación y Contabilidad, 100, 195-217.

Cardona A. M., Vera A. L. y Tabares Quiroz, J. (2008). Las dimensiones del emprendimiento empresarial: la experiencia de los programas cultura y fondo emprender en Medellín. Cuadernos de investigación (69), 64. 
Christensen, S. (2005). Enabling intrapreneurship: the case of a knowledge-intensive industrial company. European Journal of Innovation Management, 8 (3), 305-318.

Dehter, M. (2002). El ser emprendedor: modelo para desarrollar comportamiento emprendedor individual y corporativo. Recuperado el 3 de enero de 2014 de: https://www.u-cursos.cl/ingenieria/2005/1/IN505/1/ buscador/?q=ser+emprendedor.

Ecotec (2007). Design and implementation of the pilot action “ERASMUS for young entrepreneurs". Enterprise and industry. Working Paper, 2.

Etzkowitz, H. (2002). The Triple Helix of University-Industry-Government Implications for Policy and Evaluation. Working Paper Science Policy Institute, 11.

Etzkowitz, H. (2004). The evolution of the entrepreneurial university. International Journal of Technology and Globalisation, 1 (1), 64 - 77 .

Etzkowitz, H.y Leydesdorff, L. (1995). The Triple Helix University Industry Gobernment Relations: A laboratory for Knowledge based Economic Development. EASST Review, 14, 14-19.

Fontela, E., Guzmán, J., Pérez, M. y Santos, F. (2006). The art of entrepreneurial foresight. Madrid: Universidad Antonio de Nebrija.

Formichella, M. (2004). El concepto de emprendimiento y su relación con la educación, el empleo y el desarrollo local. Buenos Aires: Instituto Nacional de Tecnología Agropecuaria. Recuperado el 1 de enero de 2014 de: http://www.inta.gov.ar/barrow/info/documentos/desarrollo_local/emprendydesarrollolocal.pdf

Gámez, J. (2009). Aproximación a los modelos de emprendimiento. Management, 18 (31), 153-170.

Gibb, A. y Hannon, P. (2007). Towards the Entrepreneurial University. International Journal of Entrepreneurship Education, 4, 73-110.

Giraldo A., Bedoya G. y Vargas, C. (2009). Principales limitaciones del empresarismo que afectan el desarrollo económico y social del país. Revista EAN, 66, 99-112.

Gómez, M. P.y Satizábal, K. (2011). Educación en emprendimiento: fortalecimiento de competencias emprendedoras en la Pontificia Universidad Javeriana Cali. Economía, Gestión y Desarrollo (11), 121-151.

González, G.y González, M. (2003). Extensión universitaria: principales tendencias en su evolución y desarrollo. Revista cubana de educación superior, 23 (1), 15-26.

Gutiérrez, G. (2011). Educación emprendedora en la universidad: educando para el futuro. Retos, 2 (1), 49-68.

Guzmán, A. y Trujillo, M. A. (2008). Emprendimiento social-revisión de literatura. Estudios Gerenciales, 24 (109), 105-125.

Hisrich R., Peters, M. y Shepherd, D. (2005). Entrepreneurship. Madrid: McGraw Hill. 
Kantis, H., Postigo, S., Federico, J. y Mamborini, M. (2001). El surgimiento de emprendedores de base universitaria: ¿En qué se diferencian? Evidencias Empíricas para el Caso de Argentina. PRODEM UNGS. Recuperado el 3 de enero de 2014 de: http://www.littec.ungs.edu.ar/pdfespa\%F1ol/DT\%2006-2003\%20Kantis-PostigoFederico-Tamborini.pdf

Kirby, D. (2004). Entrepreneurship Education: ¿Can Schools Meet the Challenge? Journal Education and Training, 46 (8), 510-519.

Kunkel, S. W. (1991). El impacto de la estructura de la estrategia y de la industria en nuevo funcionamiento de la empresa. Doctoral Dissertation, University of Georgia. Dissertation Abstracts International.

Lundvall, B. (1997). National Systems and National Styles of Innovation. Manchester: Fourth International ASEAT Conference "Differences in 'styles".

Maldonado, O. (2008). Universidad, Estado e industria, del "triángulo de Sábato" al Sistema Nacional de Innovación. I Congreso Internacional de Gestión Tecnológica e Innovación realizado entre el 14 y 15 de agosto de 2008 en Bogotá D.C., Colombia.

Ministerio de Comercio, Industria y Turismo (2006). Ley 1014 de enero 26 de 2006: De fomento a la cultura del emprendimiento. Recuperado el 3 de enero de 2014 de: http://www.mineducacion.gov.co/1621/articles-94653_archivo_pdf.pdf

Ministerio de Comercio, Industria y Turismo (2009). Política de emprendimiento. Recuperado el 1 de enero de 2014 de: https://www.mincomercio.gov.co/minindustria/publicaciones.php?id=16435.

Morales, M. (2009). El clima de emprendimiento, un determinante clave en la intención emprendedora de los estudiantes de Escuelas de Negocio. Asamblea Anual Cladea 2009 -Track 1: Educación en las Escuelas de Administración, realizado en la Universidad del Pacífico, Ecuador.

Morales M. y Ortiz, C. (2011). Pasantías estudiantiles como estrategia de vinculación y transferencia de conocimiento al entorno en la Universidad Militar Nueva Granada. XIV Congreso Latino-Iberoamericano de Gestión Tecnológica realizado por la Asociación Latino-Iberoamericana de Gestión Tecnológica - ALTEC en la ciudad de Lima, Perú.

Morales, M., Pineda, K. y Ávila, K. (2011). Organizaciones innovadoras a partir de la interacción con la universidad: casos exitosos. II Simposio Iberoamericano de Estudios Gerenciales: una mirada interdisciplinar a la innovación. Cali, Colombia.

Morales, M., Plata, P.y Casallas, C. (2011). Los parques tecnológicos en Colombia como mecanismo de vinculación universidad-entorno. Revista Libre Empresa, 8 (1), 11-29.

Morales, M., Mira, G. y Arias, M. (2010). Enfoques y retos de la función de extensión universitaria como mecanismos de integración: Universidad, Empresa, Estado. II Congreso Internacional de Gestión Tecnológica e Innovación realizado en Bogotá D.C., Colombia.

Organización para la Cooperación y el Desarrollo Económicos (OCDE y Eurostat) (2006). Manual de Oslo: Guía para la recogida e interpretación de datos sobre innovación. París: OCDE. Recuperado el 1 de enero de 2014 de: http://www.uis.unesco.org/Library/Documents/OECDOsloManualo5_spa.pdf 
Ortiz, C. y Herrera, B. (2010). Formación en emprendimiento en las facultades de Administración en Bogotá. IV Workshop de Emprendimiento Universitario y V Encuentro Regional de Emprendimiento Realizado entre el 6 y 7 de mayo de 2010, Ibagué, Colombia.

Ortiz, C. y Morales M. (2011). La extensión universitaria en América Latina: concepciones y tendencias. Educación y Educadores, 14 (2), 349-366.

Ospina, M. y Sanabria, P. (2010). Un enfoque de mercadeo de servicios educativos para la gestión de las organizaciones de educación superior en Colombia: el modelo MIGME. Revista Facultad de Ciencias Económicas - Investigación y Reflexión, 18 (2), 107-136.

Pinchot, G. (2003). Intrapreneuring in action: A handbook of Business Innovation. San Francisco: Berrett-Koehler Publishers.

Pineda, K. y Morales, M. (2011). Perspectivas de la incubación de empresas de base tecnológica en las tres principales ciudades de Colombia. VI Encuentro de Investigaciones realizado por la Universidad Militar Nueva Granada, Bogotá. D.C., Colombia.

Pineda, K. y Morales, M. (2010). Integración universidad, empresa y estado: retos para la UMNG. V Encuentro de Investigaciones realizado por la Universidad Militar Nueva Granada, Bogotá. D.C., Colombia.

Pineda, K., Morales M. y Ortiz, C. (2011). Modelos y mecanismos de interacción Universidad-Empresa-Estado: retos para las universidades colombianas. Revista Equidad \& Desarrollo, 15, 41-67.

Ramírez, A. (2008). ¿Qué es el emprendimiento social? OIT y Schwab Foundation for Social Entrepreneurship. Recuperado el 3 de enero de 2014 de: http://www.comunicacionparaeldesarrollo.org/media_files/download/emprendedurismosocialq\&a.pdf

Restrepo, P. (1999). Origen de las universidades en general y de la Universidad de San Buenaventura en especial. Management, 8 (11), 15-28.

Rodríguez, A. (2009). Nuevas perspectivas para entender el emprendimiento empresarial. Revista Pensamiento y Gestión, 26 (1), 94-119.

Rofman, A. y Vázquez, J. (2006). La extensión universitaria en tiempos de crisis económico-social la experiencia de la Facultad de Ciencias Económicas de la Universidad de Buenos Aires en el lapso 2004-2006. Ciencia, docencia y tecnología, 17 (32), 9-48.

Rosa, P.y Bowes, A. (1990). Entrepreneurship: Some lessons of Social Anthropology. University of Cologne: E.C.S.B. 4th Research in Entrepreneurship.

Sábato, J. y Botana, N. (1986). La ciencia y la tecnología en el desarrollo futuro en América Latina. Bellagio: The World Order Models Conference.

Sanabria, P. (2015). La función de docencia universitaria: marco conceptual para el desarrollo de un modelo de diagnóstico estratégico e intervención de las capacidades de formación como mecanismo de integración universidad-sociedad-estado. Documento pendiente de publicación. 
ISSN 0123-1294 | Educ.Educ. Vol. 18. No. 1 | Enero-Abril de 2015 | pp. 111-134.

Universidad de La Sabana | Facultad de Educación

Sarasvathy, S. (2001). ¿What makes entrepreneurs entrepreneurial? University of Washington, School of Business. Harvard Business Review, 1-9.

Schiller, D. (2006). Nascent innovation systems in developing countries: University responses to regional needs in Thailand. Industry and innovation, 13 (4), 481-504.

Schumpeter, J. A. (2003). Capitalism, socialism and democracy. Estados Unidos: Roudledge.

Shapero, A. y Sokol, L. (1982). Some social dimensions of entrepreneurship, Encyclopedia of entrepreneurship. Englewood Cliffs, NJ: Prentice Hall.

Sutz, J. (2000). The university-industry-government relations in Latin America. Elsevier Science Research Policy, 29, 279-290.

Toca, C. (2010). Consideraciones para la formación en emprendimiento: explorando nuevos ámbitos y posibilidades. Estudios Gerenciales de la Universidad del Rosario, 6 (17), 41-6o.

Tornatzky, L., Waugaman, P. y Gray, D. (2002). Innovation U: New University Roles in a Knowledge Economy. Research Triangle Park, North Carolina: Southern Growth Policies.

Torres, A., Dutrenit, G., Becerra, N. y Sampedro, J. (2009). Patrones de vinculación academia-industria: factores determinantes en el caso de México. $4^{\circ}$ Congreso Internacional de Sistemas de Innovación para la Competitividad realizado en México.

Torres, M., Sánchez, M. y Márquez, J. (1996). Fundamentos para un modelo curricular de extensión universitaria de carácter integrador. Recuperado el 2 de enero de 2014 de: http://hosting.udlap.mx/sitios/unionlat.extension/pdf/1996/fundamentos_para_un_modelo_curricular.pdf

Universidad Militar Nueva Granada (UMNG) (2009). Proyecto Educativo Institucional (PEI). Bogotá: Universidad Militar Nueva Granada.

Universidad Militar Nueva Granada (UMNG) (2010). La proyección social: una mirada desde la UMNG. Bogotá: Universidad Militar Nueva Granada.

United Nations Educational, Scientific and Cultural Organization (Unesco) (1998). Declaración Mundial sobre Educación Superior en el Siglo XXI: visión y acción de la Unesco. Recuperado el 1 de enero de 2014 de: http:// www.unesco.org/education/educprog/wche/declaration spa.htm

Veciana, J. (2001). Creación de empresas como campo de estudio y salida profesional: evolución, estado actual y tendencias de desarrollo. RGE-Revista de Gestao e Economia, 2-91.

Vega, J. (2002). La extensión universitaria: función integradora del quehacer universitario en el presente siglo. Investigación y Desarrollo, 10 (1), 26-39.

Vesga, R. (s. f.). Emprendimiento e innovación en Colombia: ¿qué nos está haciendo falta? Observatorio de Competitividad del Centro de Estrategia y Competitividad. Recuperado el 3 de enero de 2014 de: http://cec. uniandes.edu.co/images/pdf/rav.pdf 